\title{
Near field of stacked diffraction gratings
}

\author{
Francisco Jose Torcal-Milla*, Luis Miguel Sanchez-Brea, Eusebio Bernabeu \\ Universidad Complutense de Madrid, Applied Optics Complutense Group, Optics Department, Facultad de Ciencias Físicas, Ciudad Universitaria s.n., 28040, Madrid, Spain
}

\section{A R T I C L E I N F O}

\section{Article history:}

Received 16 October 2012

Accepted 15 March 2013

\section{Keywords:}

Diffraction

Diffraction gratings

Fresnel approximation

\begin{abstract}
A B S T R A C T
We obtain a general analytical formulation for determining the near field produced by $N$ diffraction gratings disposed in stack using a scalar approximation. Parameters of the gratings such as typeamplitude/phase-, fill factors, periods and relative positions between gratings along the $x$ and $y$ axes are considered. The obtained formulation is useful for analyzing problems which involve several diffraction gratings, such as optical encoders since it is computationally faster than integral formulations. Finally, analytical results are compared with numerical simulations based on the Rayleigh-Sommerfeld equation.
\end{abstract}

(c) 2013 Elsevier GmbH. All rights reserved.

\section{Introduction}

Diffraction gratings are elements which periodically modulate one or more properties of the impinging light [1]. They can be found in numerous applications and different areas of research, such as spectroscopy, optical metrology, and Moiré interferometry [2-5]. In the near field, systems with 1 and 2 gratings have been well analyzed since they produce Talbot and Moiré effects, respectively $[6,2]$. Nevertheless, there exist optical devices which use three and even more diffraction gratings in stack as, for example, optical encoders [7-10], or other applications [11-13]. Besides, optical devices in which the beam passes through the same grating several times can also be found [14].

When the optical device has more than two gratings, or the light beam passes through the grating more than twice, the problem of determining the field after them, although simple, becomes tedious. For example, optical linear encoders with 3 diffraction gratings have been analyzed by Crespo et al. [7]. Also, a multi-grating system has been studied for Lau effect. In this case, the intensity distribution for $N$ gratings is described as a joint correlation amongst the functions of gratings [15]. The use of optical elements in stack has also been used for improving the diffractive focusing capabilities of X-rays using stacked Fresnel $\pi$-plates [16]. In these two last cases, the integrals involved cannot be solved and the results are given in an integral form. Goodman analyzes how to describe the optical propagation process in a complex optical system [17], although an analytical non-integral solution to the problem is not proposed.

\footnotetext{
* Corresponding author.

E-mail address: ftorcalmilla@fis.ucm.es (F.J. Torcal-Milla).
}

In the present work, we calculate an analytical general expression for the near-field diffracted by a number of diffraction gratings disposed in stack when they are illuminated by a monochromatic plane wave. An scalar approach has been applied, considering Fresnel approximation. The final result, obtained after solving the involved integrals, can be applied, for example, in the analysis of optical encoders. The numerical performances are greatly improved when integrals are not present into the computation.

\section{Theoretical description}

A sketch of the problem is shown in Fig. 1. A monochromatic plane wave, with wavelength $\lambda$ impinges on a system composed by $N$ diffraction gratings, separated distances $z_{1}, z_{2}, \ldots, z_{N-1}$ to each other. The observation plane is placed at a distance $z_{N}$ from the last diffraction grating. Considering Thin Element Approximation, their transmittances can be described as Fourier series expansions

$t_{j}\left(x_{j}\right)=\sum_{n_{j}} a_{j, n_{j}} \exp \left[i q_{j} n_{j}\left(x_{j}+\Delta x_{j}\right)\right], \quad j=1,2, \ldots, N$,

where $i=\sqrt{-1}, a_{j, n_{j}}$ are the Fourier coefficients for the grating $j$-th with $n_{j}$ integer, $q_{j}=2 \pi / p_{j}$, and $p_{j}$ is the period of the grating $j$-th.

The purpose of this work is to reach a general non-integral expression to describe the near field produced by a system of $N$ stacked gratings. Firstly, let us show the field produced by one diffraction grating. Considering the Fresnel formalism and plane wave illumination, the field, $U_{1}\left(x_{1}, z_{1}\right)$, at a distance $z_{1}$ from the grating can be obtained solving [18]

$U_{1}\left(x_{1}, z_{1}\right)=\frac{(1+i) \exp \left(i k z_{1}\right)}{\sqrt{2 \lambda z_{1}}} \int_{-\infty}^{\infty} t_{1}\left(x_{0}\right) \exp \left[i \frac{k}{2 z_{1}}\left(x_{1}-x_{0}\right)^{2}\right] d x_{0}$ 


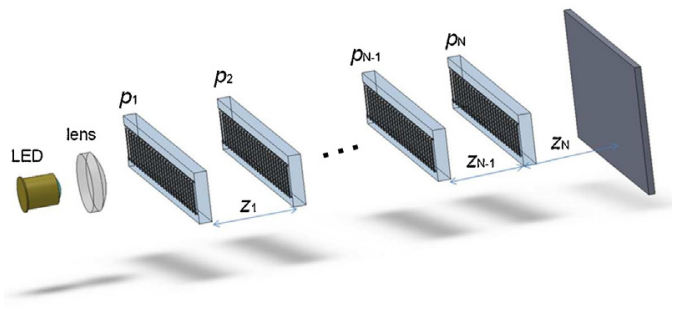

Fig. 1. Sketch of the system with $N$ stacked gratings

being $k=2 \pi / \lambda$ and $x_{0}$ the $x$-coordinate at the grating plane. This integral is easily solved[19]

$$
\begin{aligned}
U_{1}\left(x_{1}, z_{1}\right)= & \frac{\exp \left(i k z_{1}\right)}{\sqrt{\lambda z_{1}}} \sum_{n_{1}} a_{1, n_{1}} \exp \left[\frac{2 \pi i n_{1}\left(x_{1}+\Delta x_{1}\right)}{p_{1}}\right] \\
& \times \exp \left(\frac{-2 \pi i n_{1}^{2} z_{1}}{z_{T}}\right),
\end{aligned}
$$

where $z_{T}=2 p_{1}^{2} / \lambda$ is the so-called Talbot distance. The intensity after the grating is obtained from the field (Eq. (3)) just performing $I_{1}\left(x_{1}, z_{1}\right)=U_{1}\left(x_{1}, z_{1}\right) U_{1}^{*}\left(x_{1}, z_{1}\right)$

$$
\begin{aligned}
I_{1}\left(x_{1}, z_{1}\right)= & \frac{1}{\lambda z_{1}} \sum_{n_{1}, n^{\prime}{ }_{1}}^{\infty} a_{1, n_{1}} a_{1, n^{\prime}{ }_{1}}^{*} \exp \left[\frac{2 \pi i\left(n_{1}-n^{\prime}{ }_{1}\right)\left(x_{1}+\Delta x_{1}\right)}{p_{1}}\right] \\
& \times \exp \left[\frac{-2 \pi i\left(n_{1}^{2}-n_{1}^{\prime 2}\right) z_{1}}{z_{T}}\right] .
\end{aligned}
$$

Eq. (4) reveals the well known Talbot effect [6], which consists of the apparition of replications of the grating intensity pattern at integer multiples of the Talbot distance from the grating.

Now, let us calculate the field diffracted by two gratings in tandem. The second grating is placed at a distance $z_{1}$ from the first one. The field after the second grating, $U_{2}\left(x_{2} ; z_{1}, z_{2}\right)$, is obtained by multiplying Eq. (3) by the transmittance of the second grating and propagating the field to a distance $z_{2}$ from the second grating. Solving the integral, it results

$$
\begin{aligned}
U_{2}\left(x_{2} ; z_{1}, z_{2}\right)= & \frac{\exp \left[i k\left(z_{1}+z_{2}\right)\right]}{\lambda \sqrt{z_{1} z_{2}}} \sum_{n_{1}, n_{2}} a_{1, n_{1}} a_{2, n_{2}} \times \exp \left\{i \left[n_{1} q_{1}\left(x_{2}+\Delta x_{1}\right)\right.\right. \\
& \left.\left.+n_{2} q_{2}\left(x_{2}+\Delta x_{2}\right)\right]\right\} \times \exp \left(-i \frac{q_{1}^{2} n_{1}^{2} z_{1}}{2 k}\right) \exp \left[-i \frac{\left(n_{1} q_{1}+n_{2} q_{2}\right)^{2} z_{2}}{2 k}\right] .
\end{aligned}
$$

In the same fashion as the previous case, the intensity after the second diffraction grating, $I_{2}\left(x_{2} ; z_{1}, z_{2}\right)=U_{2}\left(x_{2} ; z_{1}, z_{2}\right) U_{2}^{*}\left(x_{2} ; z_{1}, z_{2}\right)$, results in

$$
\begin{aligned}
& I_{2}\left(x_{2} ; z_{1}, z_{2}\right)=\frac{1}{\lambda^{2} z_{1} z_{2}} \sum_{n_{1}, n_{1}^{\prime}, n_{2}, n_{2}^{\prime}} a_{1, n_{1}} a_{1, n^{\prime} 1}^{*} a_{2, n_{2}} a_{2, n^{\prime}}^{*} \times \exp \left[i\left(n_{1}-n_{1}^{\prime}\right) q_{1}\left(x_{2}+\Delta x_{1}\right)\right. \\
& \left.\quad+i\left(n_{2}-n_{2}^{\prime}\right) q_{2}\left(x_{2}+\Delta x_{2}\right)\right] \times \exp \left[\frac{-i\left(n_{1}^{2}-n_{1}^{\prime 2}\right) q_{1}^{2} z_{1}}{2 k}\right] \\
& \quad \times \exp \left\{\frac{-i\left[\left(n_{1}-n_{1}^{\prime}\right) q_{1}+\left(n_{2}-n_{2}^{\prime}\right) q_{2}\right]\left[\left(n_{1}+n^{\prime} 1\right) q_{1}+\left(n_{2}+n_{2}^{\prime}\right) q_{2}\right] z_{2}}{2 k}\right\} .
\end{aligned}
$$

Classical Moire effect can be explained based on the previous equation. It occurs when the period of both gratings is equal, $q_{2}=q_{1}$, $z_{1}=m z_{T}$ with $m$ integer, and the observation plane is placed just after the second grating, $z_{2}=0$ [19].

In a similar way, we can obtain the field, $U_{3}\left(x_{2} ; z_{1}, z_{2}, z_{3}\right)$, for the case of three gratings

$$
\begin{aligned}
U_{3}\left(x_{2} ; z_{1}, z_{2}, z_{3}\right)= & \frac{\exp \left[i k\left(z_{1}+z_{2}+z_{3}\right)\right]}{\lambda^{3 / 2} \sqrt{z_{1} z_{2} z_{3}}} \sum_{n_{1}, n_{2}, n_{3}} a_{n_{1}, 1} a_{n_{2}, 2} a_{n_{3}, 3} \\
& \times \exp \left\{i \left[n_{1} q_{1}\left(x_{3}+\Delta x_{1}\right)+n_{2} q_{2}\left(x_{3}+\Delta x_{2}\right)\right.\right. \\
& \left.\left.+n_{3} q_{3}\left(x_{3}+\Delta x_{3}\right)\right]\right\} \times \exp \left(-i \frac{q_{1}^{2} n_{1}^{2} z_{1}}{2 k}\right) \\
& \times \exp \left[-i \frac{\left(n_{1} q_{1}+n_{2} q_{2}\right)^{2} z_{2}}{2 k}\right] \\
& \times \exp \left[-i \frac{\left(n_{1} q_{1}+n_{2} q_{2}+n_{3} q_{3}\right)^{2} z_{3}}{2 k}\right] .
\end{aligned}
$$

Analyzing the results obtained for one, two, and three gratings, it is possible to extract a general analytical solution for the case of $N$ stacked gratings at the near field. The complex field after the $N$-th grating, at a distance $z_{N}$ from it, is given by

$$
\begin{aligned}
U_{N}\left(x_{N} ; z_{1}, \ldots, z_{N}\right)= & \frac{1}{\lambda^{N / 2}} \prod_{j=1}^{N} \frac{1}{\sqrt{z_{j}}} \sum_{n_{j}} a_{j, n_{j}} \exp \left(i k z_{j}\right) \exp \left[i n_{j} q_{j}\left(x_{N}+\Delta x_{j}\right)\right] \\
& \times \exp \left[-\frac{i}{2 k}\left(\sum_{h=1}^{j} n_{h} q_{h}\right)^{2} z_{j}\right] .
\end{aligned}
$$

Using Eq. (8), a general analytical solution for the intensity can also be obtained, $I_{N}\left(x_{N} ; z_{1}, \ldots, z_{N}\right)=$ $U_{N}\left(x_{N} ; z_{1}, \ldots, z_{N}\right) U_{N}^{*}\left(x_{N} ; z_{1}, \ldots, z_{N}\right)$, resulting in

$$
\begin{aligned}
I_{N}\left(x_{N} ; z_{1}, \ldots, z_{N}\right)= & \frac{1}{\lambda^{N}} \prod_{j=1}^{N} \frac{1}{z_{j}} \sum_{n_{j}, n_{j}^{\prime}} a_{n_{j}, j} a_{n_{j}^{\prime}, j}^{*} \exp \left[i q_{j}\left(n_{j}-n_{j}^{\prime}\right)\left(x_{N}+\Delta x_{j}\right)\right] \\
& \times \exp \left\{-\frac{i}{2 k}\left[\sum_{h=1}^{j}\left(n_{h}-n_{h}^{\prime}\right) q_{h}\right]\left[\sum_{l=1}^{j}\left(n_{l}+n_{l}^{\prime}\right) q_{l}\right] z_{j}\right\}
\end{aligned}
$$

\section{Numerical comparison}

As an example, the intensity computed with Eq. (8) after a system with 1-5 Ronchi amplitude gratings in stack is shown in Fig. 2a. The period for the gratings is $p_{j}=20 \mu \mathrm{m}$, they are separated $z_{j}=50 \mu \mathrm{m}$ along the $z$ axis and $\Delta x_{j}=0$ along the $x$ axis. The figure shows the intensity after the last grating for each system of gratings. The wavelength is $\lambda=0.5 \mu \mathrm{m}$. For the computation, diffraction orders $n_{j}=-3,-1,0,1,3$ have been considered, since they present $\approx 95 \%$ of the total energy for this kind of gratings in transmission configuration. These results are compared to those obtained with a numerical integration of the Rayleigh-Sommerfeld formula [20]. As it can be observed in Fig. $2 b$, the results are quite similar. We can see that light concentrates as the number of gratings increases, producing a kind of array illuminator, as it is shown in [21]. The differences between analytical and numerical results are due to truncations of terms in the analytical equation.

\section{Conclusions}

In this work, we obtain an analytical non-integral solution for the diffracted optical field after $N$ diffraction gratings in stack. The result is valid for the near field in scalar approach, that is, when the period of the grating is much larger than wavelength, which can be useful for the analysis of optical encoders and other optical devices where light passes through many diffraction gratings. Finally, we compute an example and compare the analytical results 
N

1

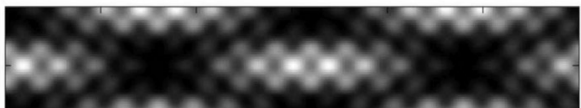

2

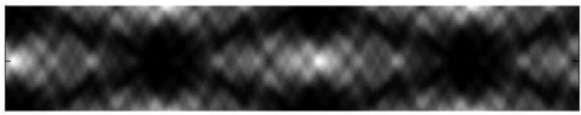

3

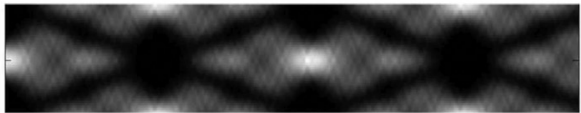

4

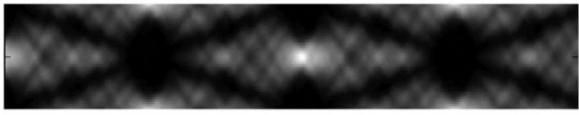

5



N

1

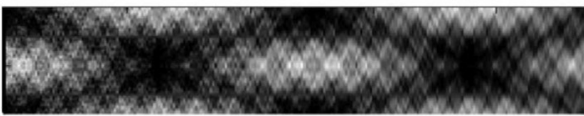

2

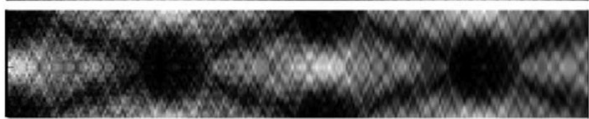

3

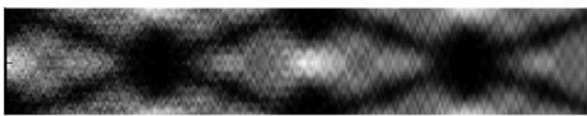

4

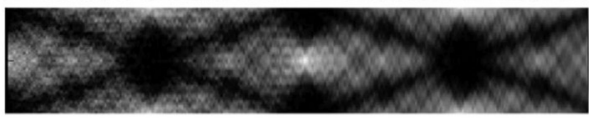

5

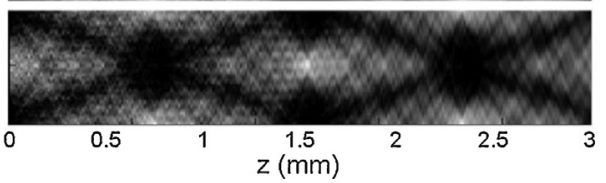

Fig. 2. (a) Analytical and (b) numerical intensity distribution at a distance $z$ from the last grating for the case of 1-5 Ronchi gratings in stack. The period for all the gratings is $p_{j}=20 \mu \mathrm{m}$, and they are separated $z_{j}=50 \mu \mathrm{m}$ along the $z$ axis and $\Delta x_{j}=0$ along the $x$ axis. The wavelength is $\lambda=0.5 \mu \mathrm{m}$. The intensity after the last grating is shown.

with those obtained by using a numerical implementation of the Rayleigh-Sommerfeld formula, showing the similarities between them.

\section{Acknowledgements}

This work has been partially supported by projects DPI201127851 and INNPACTO “FORE”, IPT-020000-2010-9 of the Ministerio de Ciencia e Innovación of Spain.

\section{References}

[1] C. Palmer, Diffraction Grating Handbook, Richardson Grating Laboratory, Eindhoven University of Technology, Eindhoven, The Netherlands, 2000.

[2] K. Patorski, Moiré Metrology, Pergamon Press, New York, USA, 1998

[3] E.G. Loewen, E. Popov, Diffraction Gratings and Applications, Marcel Dekker, New York, USA, 1997

[4] A.W. Lohmann, D.E. Silva, An interferometer based on the Talbot effect, Opt. Commun. 2 (1971) 413-415.

[5] G. Schirripa Spagnolo, D. Ambrosini, D. Paoletti, Displacement measurement using the Talbot effect with a Ronchi grating, J. Opt. A: Pure Appl. Opt. 4 (2002) S376-S380.

[6] W.H.F. Talbot, Facts relating to optical science, Philos. Mag. 9 (1836) 401-407.

[7] D. Crespo, J. Alonso, E. Bernabeu, Reflection optical encoders as three-grating Moiré systems, Appl. Opt. 39 (2000) 3805-3813.

[8] C.F. Kao, M.H. Lu, Optical encoder based on the fractional Talbot effect, Opt. Commun. 250 (2005) 16-23.

[9] C. Champenois, M. Büchner, J. Vigué, Fringe contrast in three grating MachZehnder atomic interferometers, Eur. Phys. J. D 5 (1999) 363-374.

[10] X.Zhang, Y. Huang, X. Ren, H. Huang, Q. Wang, RCE photodetector with cascaded grating structure, Proc. SPIE 7361 (2009), 7631101-76311017.

[11] F. Montiel, M. Neviere, Infrared modulation via coupling gratings lying on III-V multiple quantum wells, Opt. Commun. 138 (1997) 172-184.

[12] W. Nakagawa, R.Ch. Tyan, P.Ch. Sun, F. Xu, Y. Fainman, Ultrashort pulse propagation in near field periodic diffractive structures by use of rigorous coupled wave analysis, J. Opt. Soc. Am. A 18 (2001) 1072-1081.

[13] P. Schau, K. Frenner, L. Fu, H. Schweizer, H. Giessen, W. Osten, Design of hightransmission metallic meander stacks with different grating periodicities for subwavelength-imaging applications, Opt. Express 19 (2011) 3627-3636.

[14] G. Michel, K. Dohlen, J. Martignac, J.C. Lecullier, P. Levacher, C. Colin, Interferential scanning grating position sensor operating in space at $4 \mathrm{~K}$, Appl. Opt. 42 (2003) 6305-6313.

[15] L. Liu, Lau effect in a multi-grating system, Opt. Commun. 70 (1989) 267-271.

[16] E. Cagniot, M. Fromager, T. Godin, M. Traiche, N. Passilly, B. Paivanranta, K. AitAmeur, Cascades of $\pi$-phase plates: a transparent diffractive focusing system, J. Opt. Soc. Am. A 27 (2010) 1647-1654.

[17] J.W. Goodman, Introduction to Fourier Optics, Roberts \& Company Publishers, Greenwood Village, USA, 2005.

[18] M. Born, E. Wolf, Principles of Optics, Pergamon Press, Oxford, UK, 1991.

[19] E. Keren, O. Kafri, Diffraction effects in Moiré deflectometry, J. Opt. Soc. Am. A 2 (1985) 111-120.

[20] F. Shen, A. Wang, Fast-Fourier-transform based numerical integration method for the Rayleigh-Sommerfeld diffraction formula, Appl. Opt. 45 (2006) 1102-1110.

[21] V. Arrizon, J.G. Ibarra, A.W. Lohmann, Array illuminators with phase gratings in cascade, Opt. Commun. 124 (1996) 229-234. 